\title{
Educación superior, el futuro del trabajo y la automatización
}

\section{Resumen}

La educación es factor fundamental para lograr mejorar las condiciones de vida. El crecimiento de cobertura en educación superior no es simétrico al empleo profesional. Sobre la pertinencia laboral incide el dinamismo de la cuarta revolución industrial que transforma los roles, sustituyendo el trabajo humano por robots y algoritmos "inteligentes" capaces de tomar decisiones. Además de competencias técnicas, acordes con avances tecnológicos, se requiere nueva alfabetización digital que incluya capacidades cognitivas como pensamiento crítico y analítico que enfrente amenazas de información sesgada, prejuiciadas y dogmática que circula en la web que produce desigualdad, ignorancia y riesgos a la democracia.

Palabras clave: Pertinencia; Robotización; Alfabetización digital; Pensamiento crítico; Amenazas sociales.

\section{Abstract}

Education is a fundamental factor in improving living conditions. The growth of coverage in higher education is not symmetrical to professional employment. The dynamism of the fourth industrial revolution that transforms the roles, replacing human work with robots and "intelligent algorithms" capable of making decisions, has an impact on labor relevance. In addition to technical skills, in line with technological advances, new digital literacy is required that includes cognitive abilities such as critical and analytical thinking that face biased, prejudiced and dogmatic information threats circulating on the web that produce inequality, ignorance and risks to democracy.

Keywords: Relevance; Robotization; Digital literacy; Critical thinking; Social threats.

POR ENRIQUE GRAUE, JAIME MARTUSCELLI, CARLOS MARTÍNEZ LEYVA. Rector de la Universidad Nacional Autónoma de México, (UNAM). / Coordinador de Programas Académicos de la Rectoría UnAM. / Asesor de la Coordinación de Programas Académicos de la Rectoría UNAM. 


\section{Introducción}

Durante las últimas década hemos sido testigos de una transformación en los procesos productivos y tecnológicos sin precedentes en la historia, que por su impacto general y por el dinamismo que presenta en diversas áreas del conocimiento y de la innovación, desafían a los sistemas educativos para transmitir las competencias pertinentes en la formación de recursos humanos que los cambios en la estructura laboral exige hoy y demandará en el futuro. Es de sobra conocido que la educación es un factor fundamental para el avance social, pues aumenta las posibilidades económicas, promueve la movilidad social, incrementa la capacidad de tomar decisiones eficaces, mejora la calidad de vida y facilita el adecuado funcionamiento de las instituciones en una sociedad democrática. Aquellas economías que logran mejores niveles educativos y, en consecuencia, de formación de recursos humanos calificados, así como mayores capacidades de investigación, crecen más rápido que aquellas que padecen bajos niveles de cobertura y mantiene altos índices de analfabetismo.

En la sociedad del conocimiento global, el avance científico y tecnológico tiene una aceleración nunca vista, convirtiéndose en la fuerza productiva que ha impulsado la competitividad económica y transformado importantes esferas de la acción social. Se expresa en campos específicos como la comunicación en tiempo real, la automatización de procesos productivos, la modificación genética de plantas y animales, el uso de recursos biotecnológicos, la forma de atender condiciones de salud y el mayor conocimiento sobre los procesos cognitivos y de aprendizaje que han impulsado la inteligencia artificial, entre otros variados desarrollos.

Para beneficiarse de los resultados del desarrollo del conocimiento y de la innovación es necesario que la población tenga las capacidades de comprenderlas y utilizarlas en su vida cotidiana. Éste no es el caso de México, donde persiste la pobreza y la ignorancia, y un importante sector de la sociedad es excluida del derecho a la educación. De acuerdo con el Instituto Nacional de Estadística y Geografía (INEGI, 2017) existen hoy más de 5 millones de analfabetas y más de 32 millones de individuos con rezago educativo. Esto, a pesar de los esfuerzos para incrementar la cobertura, que ha alcanzado ya el 97 y 93 por ciento en enseñanza primaria y secundaria, respectivamente; de que sea de 84.6 por ciento en educación media superior (Ortega 2018) y de 37 por ciento en el nivel superior. Pero aún persiste el abandono escolar, que en secundaria alcanza 
el 28 por ciento y en el bachillerato el 50 por ciento de los inscritos. Este último nivel es particularmente preocupante, pues corresponde a una etapa en la que los jóvenes deciden su proyecto profesional y de vida. Es el tiempo en que desarrollan las competencias y habilidades que los capacitará para desempeñarse satisfactoriamente en los estudios superiores, en actividades laborales y, en general, para vivir armónicamente en sociedad. Se destaca la obligatoriedad de este nivel para el ciclo 2021-2022.

En las siguientes páginas hacemos una reflexión general sobre el crecimiento de las tasas de cobertura de la educación superior, la pertinencia o correspondencia entre la formación profesional y la incorporación de egresados a puestos laborales, y el impacto que la automatización tiene sobre los roles profesionales. Asimismo, se señalan las propuestas para desarrollar las competencias de una nueva alfabetización en el contexto de la robotización y los algoritmos, tanto las vinculadas al manejo de las tecnologías de la información y comunicación, como aquellas que promuevan el desarrollo de capacidades cognitivas personales que permitan a los usuarios enfrentar los riesgos y las amenazas que esos recursos traen consigo. Todo ello se convierte en un desafío para las instituciones educativas en cuanto a su responsabilidad de formar personas críticas, responsables y con sentido ético.

\section{Educación superior y empleo}

De acuerdo con la ANUIEs (2018) el desarrollo de la educación superior en México ha tenido dos características o tendencias distintivas: la primera es una continua expansión de la cobertura que se aprecia en el incremento constante de la matrícula. La segunda es una amplia diversificación de la oferta en varios tipos de instituciones públicas como universidades e instituciones federales; universidades públicas estatales, institutos tecnológicos del Tecnológico Nacional de México, universidades politécnicas y tecnológicas, universidades interculturales y centros públicos de investigación. Además, las 2,140 instituciones de carácter particular se diversificaron, cubriendo la tercera parte de la matrícula total. Esas características han traído como consecuencia la desconcentración y descentralización de la oferta y la multiplicación de opciones educativas beneficiando a algunas entidades federativas, pero manteniendo una brecha nunca aceptable entre diversos Estados de la República.

La ampliación de las oportunidades de acceso a la educación superior ha logrado resultados favorables en los últimos años. Considerando las modalidades escolarizada y no escolarizada, la matrícula de licenciatura del ciclo 2000-2001 fue de 2.2 millones de estudiantes; para 2010-2011 se alcanzó la cifra de 3.3 millones y para el ciclo 2016-2017 se registraron más de 4.4 millones. Este crecimiento significó la creación de 139 mil nuevos lugares cada año. La tasa bruta de cobertura pasó de 20.4 por ciento en 2000 a 37.3 por ciento en 2017 (Tuirán, 2018).

Adicionalmente, se apoyó la enseñanza no presencial o no escolarizada como las modalidades mixtas, abierta, a distancia y virtuales que han crecido de forma importante. En el año 2000 estas modalidades tenían 150 mil estudiantes, para el ciclo 2016-2017 registraron la cifra de $667 \mathrm{mil} ; 572 \mathrm{mil}$ de licenciatura que es el 14 por ciento de la matrícula y 95 mil de posgrado que significa el 28.5 por ciento de los inscritos en este nivel (Tuirán, 2018).

Sin embargo, debemos aceptar que tenemos un problema respecto a la calidad de la educación y de sus egresados. La incorporación al empleo de los egresados de la educación superior continúa siendo un serio problema. Si bien la educación es condición necesaria para mejorar el bienestar social, hoy en día la cobertura educativa por sí misma ya no es una vía segura para el empleo ni un medio para construir una perspectiva de vida mejor, pues cada vez se observa con mayor preocupación una elevada desocupación en jóvenes y, lo más lamentable, es el desempleo de aquellos con altos niveles educativos. Datos proporcionados por el Consejo Nacional de Evaluación (CONEVAL, 2018), indican que sólo el 54 por ciento de los jóvenes que estudiaron una licenciatura se encuentran ocupados y de estos, la mayoría se encuentra en condiciones precarias de seguridad social. El INEGI (2017) ha señalado que de los 3.7 millones de subocupados en el primer semestre de 2017, poco más de un millón contaban con educación media superior o superior. Según la Encuesta Nacional de Empleo, en el segundo semestre de 2016, seis de cada diez jóvenes trabajaban en empleos informales con salarios bajos, inestabilidad laboral y sin presta- 
ciones básicas como servicios de salud y seguridad social (INEGI, 2018).

La Organización para la Cooperación y el Desarrollo Económico (OCDE, 2019) señaló que en México, a pesar de que los egresados del nivel superior llegan a ganar 78 por ciento más en promedio que los que sólo tienen estudios de nivel medio, aquellos que trabajan en ocupaciones que no requieren calificaciones de educación superior o en el sector informal no se benefician de la prima salarial asociada al título universitario.

La OCDE (2019) indica también la existencia de un sesgo de género en la incorporación al empleo, dice:

Aunque las mujeres representan el 53\% de los egresados con un primer título de educación superior, más de una de cada cinco no participa en el mercado laboral. Su tasa de inactividad es tres veces mayor que la de los egresados varones, $21.3 \%$ frente a $6.9 \%$ y su tasa de ocupación es inferior $74.2 \%$ frente a $87.9 \%$.

Ello puede obedecer a prácticas culturales, discriminatorias, o a decisiones personales, pero en todo caso, es un enorme potencial desaprovechado.

En lo que se refiere a la formación en la educación superior por campo de estudio, esta Organización informa que la matrícula se concentra en unas cuantas carreras. Así, el 35 por ciento está concentrado en derecho y administración, frente al 23 por ciento del promedio de la OCDE; 24 por ciento en ingeniería, industria y construcción, frente a 17 por ciento de la OCDE; 10 por ciento en salud y bienestar, frente a 13 por ciento de la OCDE; 3 por ciento en ciencias naturales, matemáticas y estadística, frente a 6.5 por ciento de la OCDE; y tecnologías de la información y comunicación 1.9 por ciento, frente a 4.6 por ciento de la OCDE. Se hace el comparativo con la oCDE porque México es un país miembro de esa Organización.

Como es obvio, la mayoría de los egresados son de las áreas que tienen más estudiantes y además son quienes tienen mayores tasas de ocupación, pero su desempeño se lleva a cabo en una gran variedad de puestos de trabajo, muchos de los cuales no requieren la formación que cursaron. Se estima que en administración de empresas y derecho existe una tasa de sobre cualificación del 56 por ciento y en ingeniería y construcción del 53 por ciento. Esto hace pensar que para los jóvenes la posibilidad de obtener un empleo al egresar del nivel superior refuerza la tendencia a favorecer la matrícula en esos campos disciplinarios.

En 2017, el 77 por ciento de los jóvenes mexicanos egresados de licenciatura se emplearon en cuatro sectores: servicios sociales contrataron al 31 por ciento; servicios profesionales, financieros y corporativos al 18 por ciento; comercio al 15 por ciento, e industria manufacturera al 13 por ciento. La OCDE (2019) también nos dice que: "Los egresados jóvenes de programas de salud y bienestar, educación y ciencias naturales, matemáticas y estadística son particularmente proclives a iniciar sus trayectorias profesionales en el sector informal". Al mismo tiempo, hay sectores como minería y extracción, construcción, comunicaciones y transportes, que reportan dificultades para cubrir vacantes aduciendo falta de competencia y experiencia para el perfil del puesto de los aspirantes que se presentan.

\section{Automatización de roles laborales}

Existe una pertinencia débil entre la formación educativa superior y el mercado del trabajo. Este desfase se está acelerando por el ritmo de avances tecnológicos como las referentes a la robotización, la inteligencia artificial, la impresión 3D, la biotecnología, el internet móvil de alta velocidad, la adopción del análisis Big Data, la tecnología de nube, y el aprendizaje automático. Estos factores, están redefiniendo las competencias y necesidades de formación profesional que están transformando la estructura del empleo y refuerzan la tendencia a sustituir el trabajo humano por maquinas automatizadas y algoritmos con la intención de lograr mayor productividad y ganancia en la industria y, en general, en la de todo tipo de empresas. Este proceso se le conoce como la Cuarta Revolución Industrial.

De acuerdo con la Organización Internacional del Trabajo (OIT, 2017), a medida que se automaticen los procesos de trabajo se perderán aquellos empleos que impliquen tareas repetitivas y rutinarias, afectando tanto a los de manufactura, como a los empleos de oficina. De acuerdo con el Foro Económico Mundial de Davos (2018) para el año 2020 desaparecerán cinco millones de puestos de trabajo en las 15 economías más desarrolladas del mundo. En la región de Iberoamérica, 
según la Secretaria General Iberoamericana, Rebeca Grynspan (2018), esta Cuarta Revolución Industrial traerá como consecuencia que en el mundo desarrollado, entre un tercio y la mitad de todos los empleos podrán ser automatizados en los próximos 25 años. En América Latina donde los trabajos suelen ser más intensivos en mano de obra, la cifra de automatización y pérdida sería más alta, pues el impacto de la tecnología e innovación se da de manera diferente en cada país.

En un reciente estudio realizado por investigadores de la UNAM, se concluyó que México el riesgo de que el empleo sea automatizado es de 63 por ciento, debido a, entre otras causas, la creciente introducción de equipos que se ha facilitado por la caída de los precios de los robots. Esto afectaría a los trabajadores menos calificados que son los más vulnerables. El efecto más grave se daría en industrias como la automotriz, alimentaria, vestido, productos metálicos y equipo de transporte entre otros (Minian y Martínez, 2018).

Sin embargo, algo muy importante que hay que destacar es que no sólo los trabajos rutinarios y repetitivos son susceptibles de automatización, sino también muchos de los que emplean capacidades cognitivas que antes se consideraban exclusivamente "humanas". Según Harari (2016), ya en 2004, Frank Levy del Instituto Tecnológico de Massachusetts y Richard Murnane de la Universidad Harvard daban a conocer una lista de trabajos con mayor probabilidad de automatización en los Estados Unidos, y en aquel entonces descartaban algunos, como por ejemplo, los conductores de camiones que requerían de "inteligencia humana" para conducir con seguridad. Como es sabido, para el año de 2014 ya existían vehículos auto conducidos hechos por Tesla y Google que lograron integrar y operar algoritmos complejos con grandes volúmenes de datos para obtener respuestas inteligentes o decisiones oportunas, tomadas por los mismos equipos autómatas.

Los algoritmos, recordemos, son conjuntos ordenados y lógicos de operaciones sistemáticas, con reglas bien definidas que se utilizan para hacer cálculos, resolver problemas y alcanzar decisiones. Son métodos poderosos que integran información de diversa índole, siguen instrucciones predeterminadas y ofrecen solución a un tipo específico de problemas.

Minian y Martinez (2018) refieren a los investigadores de Oxford Carl Benedick y Michael A. Osborne quienes publicaron en 2013 el informe El Futuro del trabajo, en la que exploraron la probabilidad de que diferentes profesiones quedaran a cargo de algoritmos informáticos en los siguientes veinte años. Según sus estimaciones, concluyeron que el 47 por ciento de los puestos de trabajo de Estados Unidos corrían un riesgo elevado de ser ocupados por algoritmos. Señalaron que existían algunos empleos cuya sustitución era difícil en virtud de que exigen conocimiento muy específico y refinado, y sobre los cuales la automatización no produce beneficios económicos. Los autores del informe destacaron el potencial del impacto de las tecnologías sobre las ocupaciones y la probabilidad de que sean sustituidas, dado que ya se cuenta con la capacidad técnica para ello, pero no se pronunciaron por la certeza de que ello ocurra necesariamente en un plazo determinado, pues hay factores económicos, políticos, sociales, como la lucha de organizaciones laborales, que pueden retrasar este proceso. Hoy muchos sindicatos han emprendido acciones para la defensa del trabajo y para obtener seguros de protección económica por la amenaza de desplazamiento laboral. Asimismo, tampoco se tiene la certeza de que ocurra un desempleo masivo, pues sin duda surgirán nuevas profesiones o reconfiguración de los perfiles profesionales o de puestos de trabajo.

No obstante, la perspectiva de la automatización del trabajo es inminente. El Foro Económico Mundial de Davos en el informe sobre El futuro del trabajo, ya citado, el cual fue resultado de una amplia consulta mundial con empresarios, industriales, directores generales de empresas y responsables de recursos humanos, concluye que en el corto plazo (cuatro años), las máquinas incrementarán su participación en la estructura del trabajo, modificando la relación con los seres humanos. De acuerdo con ese reporte, en 2018 un promedio de 71 por ciento del total de horas de trabajo, en las industrias encuestadas, estaban a cargo de seres humanos, en comparación con el 29 ciento de las máquinas. Para el año 2022, se espera que este promedio haya cambiado a 58 por ciento de horas de trabajo realizadas por humanos y 42 por ciento por máquinas. En tareas específicas se espera que las máquinas y algoritmos aumenten en 2022 su contribución, por ejemplo; las máquinas realizarán el 62 por ciento del procesamiento de datos y la búsqueda, organiza- 
ción y transmisión de información, en comparación con el 46 por ciento actual. Incluso aquellas tareas de trabajo que hasta ahora han permanecido abrumadoramente humanas, como comunicación e interacción que tiene 23 por ciento hecho por máquinas llegará al 30 por ciento; coordinación, desarrollo, gestión y asesoramiento con 20 por ciento actual alcanzará el 29; así como el razonamiento y la toma de decisiones con el 18 por ciento hoy llegará al 27 e irán creciendo paulatinamente.

La carrera por la automatización entre los países, estimulado entre otros factores, por el abaratamiento de los equipos, ha acelerado de forma impresionante la demanda de robots industriales, incrementando la inversión en tecnologías e incidiendo en la competencia mundial. De acuerdo con la Federación Internacional de Robótica (2018) el número de robots en operación en el mundo fue de dos millones 400 mil unidades en 2018 y será de tres millones 218 mil unidades en 2020. El crecimiento de la venta de estos equipos fue como nunca entre 2012 y 2017, incrementándose a un promedio anual del 19 por ciento. Hay cinco países que concentran el 73 por ciento del volumen global de robots: China, Japón, Corea del Sur, Estados Unidos y Alemania. China ha sido el más grande mercado de robots en el mundo, manteniendo un crecimiento muy dinámico; sólo en 2017 adquirió 137 mil 900 unidades, 59 por ciento más que el año anterior y este año planea adquirir 210 mil, cantidad considerablemente mayor al volumen que instalarán Europa y América juntos.

Para poder comparar el nivel de robotización que han alcanzado los países, la Federación Internacional de Robótica define la densidad como el número de robots por cada 10 mil trabajadores manufactureros. De esa manera ubica a Corea como el país de mayor densidad con 710 robots por cada 10 mil empleados, seguido de Singapur con 658 robots, Alemania con 322, Japón con 308, Estados Unidos 189. China tuvo en 2017 una densidad 97 unidades, pero en 2009 sólo tenía 11 unidades instaladas por cada 10 mil trabajadores. El promedio mundial es 74 robots.

México se ubica en el puesto 30 con una densidad de 36 robots por cada 10 mil trabajadores, muy por abajo de la media internacional, pero se ha convertido en importante mercado emergente para la industria de robots, mientras que Brasil y Argentina tienen 20 cada uno. Esto significa que la transformación de los procesos laborales en nuestro país será más rápida que en el resto de Latinoamérica.

Las cinco industrias que más han automatizado sus procesos de trabajo a nivel mundial son la automotriz, seguida de la eléctrica y electrónica, metalmecánica, plásticos y productos químicos, y alimentos. De esa forma, la automatización está transformando rápidamente los roles laborales y las profesiones dejando a algunos francamente obsoletos y exigiendo de otros una recualificación o reentrenamiento. El reporte ya comentado del Foro Económico Mundial de Davos señala que las grandes empresas del mundo consideran que muy pronto serán casi innecesarios o redundantes roles como los desempeñados por empleados de contabilidad y nómina, gerentes de administración y servicios comerciales, contadores y auditores, empleados del servicio postal, analistas financieros, cajeros y empleados de taquillas, agentes de ventas, empleados de estadísticas, finanzas y seguros, empleados de vigilancia y seguridad, así como los abogados, entre otros empleos de los llamados de cuello blanco.

Actualmente son muy conocidas y usadas algunas aplicaciones cognitivas, como el aprendizaje automático e inteligencia artificial, las de tareas específicas como el reconocimiento e identificación de imágenes y rostros, dispositivos de ahorro de energía, máquinas de atención al cliente, análisis de movimientos bursátiles, automóviles autónomos, optimización de rutas, análisis de ponencias jurídicas y detección de enfermedades y recomendaciones terapéuticas, que incluso ya están siendo personalizadas.

Hoy ya existen potentes algoritmos informáticos como el llamado Watson de IBM, con capacidad de diagnosticar padecimientos como el cáncer de pulmón, con una certeza del 90 por ciento, teniendo una precisión mayor al diagnóstico del médico. Las tomografías computarizadas y mamografías son hoy por hoy analizadas por algoritmos especializados, que proporcionan al médico evidencia segura; se estima que Watson muy pronto pueda extender su uso en una gran cantidad de padecimientos tanto en diagnóstico, como en tratamiento. Otro ejemplo relevante es el caso la empresa Pathway Pharmaceuticals que usa el algoritmo llamado OncoFinder para seleccionar y evaluar terapias personalizadas contra el cáncer (Harari 2016: pag. 354). 
También en el campo de la medicina se cuenta con el sistema robótico Da Vinci para realizar procedimientos quirúrgicos complejos mediante cirugía de invasión mínima. La cirugía robótica se utiliza actualmente en urología, oncología, otorrinolaringología, cirugía bariátrica y de tórax. El cirujano realiza la cirugía sentado en una consola teniendo en todo momento una visión tridimensional del campo quirúrgico, manejando la plataforma digital con mayor precisión que su propia mano. De acuerdo con Gómez (2019) En el mundo existen alrededor de 3 mil 600 robots de este tipo, dos terceras partes están en Estados Unidos y el resto en Europa, en México existen, actualmente, diez.

Los abogados se encuentran sorprendidos al ver el crecimiento en servicios de asesoría jurídica automatizada manejadas por ingenieros con asesoría de abogados. El investigador del Instituto de Investigaciones Jurídicas de la UNAM. Miguel Carbonell (2019) nos informa que:

Empresas como IBM están desarrollando programas de asistentes jurídicos que tienen la capacidad de procesar millones de documentos jurídicos en pocos minutos, que sirven incluso para hacer estimaciones sobre probabilidades de éxito en determinados tipos de litigios.... [Actualmente existe] el sistema automatizado de solución de controversias de eBay que resuelve siete veces más disputas que todo el sistema judicial de Estados Unidos, sin que tenga que intervenir, al menos en la etapa inicial, ningún ser humano.... [Así que] el trabajo de los abogados va a cambiar de manera radical en los próximos años.

\section{Áreas de formación de competencias}

Estos hechos también provocan el surgimiento de nuevas demandas de trabajadores calificados, y las empresas evalúan estrategias tales como contratar personal completamente nuevo que domine las nuevas tecnologías, o sustituir a los empleados automatizando la mayoría de sus procesos, o bien, volver a capacitar a los empleados que actualmente son sólo complemento de la tecnología, liberándolos de la obligación de realizar tareas rutinarias y repetitivas. Es ya un hecho la configuración de una demanda creciente en recursos humanos calificados en áreas como el análisis de datos y resultados científicos, desarrolladores de software y aplicaciones, especialistas en comercio electrónico, especialistas en inteligencia artificial y aprendizaje automático, especialistas en Big Data, expertos en automatización de procesos, analistas de seguridad informática, diseñadores de interacción de máquinas y expertos en robótica (Serrano, 2018).

Así, aquellas tareas complejas que demanden competencias cognitivas elevadas, capacidades interpersonales y creatividad ganarán espacio. Las actividades ligadas a la investigación y desarrollo, ingenierías, matemáticas, educación, cuidados personales, entre otras, tendrán amplias oportunidades. La OIT (2017) propone a las instituciones de educación superior como opción ofrecer formaciones generales en vez de formaciones profesionales especializadas a los estudiantes, lo que mitigará el riesgo de otorgar una formación que pueda cambiar o desaparecer en el mediano plazo. Es necesario que las universidades sean innovadoras, con suficiente capacidad para mantener el ritmo de los avances científico-tecnológicos, se adapten rápidamente a la naturaleza cambiante de los empleos y sus egresados gocen de la pertinencia requerida.

Además de las competencias técnicas, el Foro Económico reconoce, asimismo, el incremento de la demanda de empleos con habilidades "netamente humanas" como pensamiento analítico, aprendizaje activo, innovación, creatividad, originalidad, persuasión, negociación, inteligencia emocional, resiliencia, entre otras, que serán relevantes en el trabajo del futuro.

Las competencias técnicas y las habilidades humanas deben contemplarse juntas en las estrategias y medios para la formación de las actuales y futuras generaciones. El reto ha sido cómo armar una estructura de enseñanza- aprendizaje pertinente y de calidad, que responda a las necesidades sociales y económicas, ante las exigencias tecnológicas, al tiempo que propicie la formación de personas íntegras, responsables y comprometidas con su comunidad.

De allí que sea importante indagar sobre el proceso educativo más pertinente para ese contexto y comparar las diversas visiones pedagógicas, así como las evaluaciones prospectivas sobre los futuros posibles donde los egresados de las universidades se desem- 
peñarán laboralmente. Al respecto hay que señalar que existen iniciativas relevantes para vincular esos aspectos. Los investigadores de la Universidad Harvard, Reimers y Chung (2016), en su trabajo Enseñanza y Aprendizaje en el Siglo XXI nos informan de algunos esfuerzos llevados a cabo en ese sentido, como el estudio enGauge publicado en 2003 en Estados Unidos patrocinado por el North Central Regional Educational Laboratory y la consultoría educativa Grupo Metiri en el que se describe el impacto de la tecnología sobre el trabajo y proponen transformar la educación para desarrollar una nueva alfabetización identificando cuatro campos de competencias:

Alfabetización Digital que comprende la básica, científica, económica, tecnológica, visual, multicultural y conciencia global;

Pensamiento inventivo o creativo que integra la adaptabilidad, manejo de la complejidad, dirección personal, curiosidad, toma de riesgos y razonamiento sensato;

Comunicación efectiva, que se refiere a trabajo en equipo, colaboración y habilidades interpersonales, responsabilidad personal, social y cívica;

Alta productividad que comprende acciones sobre priorizar, planificar y administrar para lograr resultados; uso efectivo de herramientas del mundo real y crear productos relevantes.

Posteriormente, el Foro Económico Mundial llevó a cabo un análisis de las brechas que existen entre los países para conseguir, a través de la educación, las habilidades necesarias para este siglo en un entorno laboral con creciente tecnificación y las agrupa en tres amplios apartados que son:

Las que denomina Alfabetizaciones fundamentales como matemática, científica, tecnologías de la información y comunicación, financiera, cultural, cívica y dominio de la lengua;

la promoción de Competencias, tales como pensamiento crítico, creatividad, comunicación, colaboración y solución de problemas, $\mathrm{y}$;

El cultivo de las llamadas Cualidades de carácter, referidas a la curiosidad, iniciativa, persistencia, adaptabilidad, liderazgo y conciencia social.

Las determinaciones de esas competencias y habilidades están dadas, en gran medida, por la percepción que los autores tienen sobre el presente en lo econó- mico y social, así como por las expectativas sobre los cambios en la estructura del trabajo y las necesidades futuras de las industrias y negocios. La cuestión que el Foro no aborda es la manera en que ellas puedan articularse en una estructura académica que contemple la evolución cognitiva de los estudiantes y su desarrollo personal en sus diferentes etapas de crecimiento.

Reimers y Chung (2016) también resumen los resultados de un informe del Consejo Nacional de Investigación de los Estados Unidos, coordinado por Margaret Hilton y James Pellegrino que con el título Educación para la vida y el Trabajo: generar conocimiento $y$ habilidades transferibles en el siglo XXI intentaron, mediante investigación psicológica, identificar aquellas habilidades que tienen mayor impacto en los individuos durante su desarrollo personal y las competencias asociadas al trabajo, así proponen que las instituciones educativas centren la formación de sus estudiantes promoviendo el desarrollo de:

Competencias cognitivas que incluyen el pensamiento crítico, solución de problemas, análisis, razonamiento y argumentación, aprendizaje flexible, toma de decisiones y ejecución. Asimismo, conocimiento o dominio de información, identificación de sesgos y prejuicios en las fuentes que se consulten, manejo de las tecnologías de la información y comunicación y capacidad de comunicación oral y escrita.

Competencias personales, referidas a una apertura intelectual para la adaptabilidad, flexibilidad, apreciación artística y cultural, apreciación de la diversidad, disposición para el aprendizaje continuo y curiosidad intelectual, responsabilidad ética, integridad, conciencia del campo laboral, profesionalismo, asunción de la ciudadanía, autorregulación y autoevaluación y salud física y psicológica, y;

Competencias interpersonales tales como trabajo en equipo, cooperación, empatía, solución de conflictos, negociación, liderazgo, comunicación asertiva y confianza en los demás.

Como se observa, la identificación de las competencias necesarias para el presente siglo propuestas por investigadores e instituciones son muy coincidentes, especialmente las referidas a las personales e interpersonales. La última propuesta de competencias se asemeja más a lo que se considera como la formación integral de los estudiantes al considerar la cuestión 
artística y cultural, salud, civismo, ética, aceptación de la diversidad y solidaridad. Consideramos que éstas serían implementadas como actividades transversales aplicables en todos los campos profesionales. A cada habilidad enunciada se requiere dotarle de contenido concreto de manera que permita establecer objetivos de aprendizaje y expectativas que puedan ser compartidos por los diferentes actores educativos y pueda evaluarse el nivel de logro de cada una de ellas.

\section{Amenazas de la automatización}

En general, la organización escolar está actualmente más orientada a la enseñanza de los aspectos cognitivos que a impulsar las competencias personales e interpersonales, mismas que no se consideran en las evaluaciones escolares, ni de estudiantes ni de programas. No obstante, la formación de competencias tales como el análisis y razonamiento, pensamiento crítico, capacidad de discernimiento de la información, formación ética y apertura intelectual son cada vez más relevantes, y se debe asegurar su incorporación al repertorio conductual de los estudiantes, ya que les permitirá enfrentar los riesgos que la automatización, la inteligencia artificial, y el aprendizaje automático, entre otros avances, traen consigo.

Hoy la transmisión de información de los contenidos de asignaturas que integran un currículum escolar es insuficiente, o mucha de ella ya no es necesaria. La información está disponible para cualquiera que tenga acceso a Internet. Lo que se espera es que se tenga la orientación para distinguir la forma en que operan los espacios en línea, distinguir la información fundada en investigación científica y aquella que no lo es, de comprender y separar lo importante de lo accesorio y de la que le permita formarse una visión holística de la realidad, pensar por sí mismos, descartando aquella información que está fundada en prejuicios y dogmas políticos o religiosos (Harari, 2018).

Aunque nos maravillemos de los avances tecnológicos y de la forma en que pueden mejorar la vida de los seres humanos, también traen aparejados peligros para los estudiantes y para la sociedad en su conjunto. Al respecto, Bhatt y MacKenzie (2018) han advertido sobre el desafío que enfrenta la educación superior por la alfabetización digital y el deber de enseñar a los estudiantes sobre la forma en que funcionan las plataformas en línea, de manera que se evite y contrarreste "la construcción de la ignorancia" a partir del poder de los algoritmos para producir, mantener y difundir conocimientos sin sustento científico y que son aceptados, porque a la información que circula por Internet se le atribuye autoridad epistémica. Lo preocupante, afirman, es que escuelas y bibliotecas están siendo desplazadas por herramientas de la web porque los usuarios consideran que son recursos públicos que están libres de intereses comerciales y sesgos valorativos, lo que no necesariamente es el caso. Ellos dicen:

¿Por qué debería ser esto una cuestión de preocupación? Una de las razones es que los algoritmos están creando «nuevas asimetrías de poder», y se perciben como conocedores de nosotros mismos mejor que nosotros La minería de datos, es un ejemplo útil. La interpretación y el procesamiento de los datos realizan una serie de correlaciones a través de las cuales los intereses de los usuarios se individualizan para anticipar acciones futuras. Estos perfiles predictivos son ingredientes esenciales para las estrategias de mercadeo en línea, y de los cuales los usuarios pueden no tener conocimiento. Hemos aprendido que nuestras identidades son objetos virtuales que las empresas pueden comprar y vender sin que lo sepamos, o sin que nuestras voces sean escuchadas o tomadas en cuenta. La forma en que buscamos, usamos y comunicamos información a través de la web y los roles y efectos de los motores de búsqueda ha sido, y sigue siendo, en gran parte desconocida para la mayoría de los usuarios... Dado que los procedimientos algorítmicos están determinados por los propietarios de las plataformas de acuerdo con sus intereses, se puede crear un perfil de usuario utilizando algoritmos de personalización, mediante la recopilación y almacenamiento de pistas según el historial de navegación, las direcciones de Internet Protocolo (IP), la actividad de las redes sociales, el contenido del correo electrónico y palabras clave. No solo los usuarios pueden ignorar lo que está sucediendo, sino que también pueden no saber cuáles son sus derechos 
o los usos para los cuales se está utilizando esta extracción pasiva de datos. Como entendemos cada vez más, tal ignorancia no es una brecha benigna para el conocimiento

Las plataformas de Internet contienen motivaciones, valores e intereses que están presentes desde su concepción. Los códigos y sistemas de inteligencia artificial pueden enmascarar, reforzar y profundizar la desigualdad social; los algoritmos no son todos benignos, neutrales u objetivos, pues los propios diseñadores pueden inducir al prejuicio racial, sexismo y misoginia, y transmitir los valores e intereses de los patrocinadores. Así lo reconoció el excolaborador de Google, Guillaume Chaslot, experto en inteligencia artificial al explicar cómo funcionaba el canal de videos YouTube, a decir de Nuño Domínguez, (2018):

El canal de video online gana un céntimo de euro por cada hora. Cada día en todo el mundo se ven unas 1000 millones de horas, con lo que se gana mucho dinero. El algoritmo que recomienda otros videos después de una reproducción tiene como principal objetivo maximizar el tiempo de permanencia. Lo que sucede es que el algoritmo acaba recomendando videos sobre teorías de la conspiración, discursos de odio, racistas o sexistas, porque esos son los que tienen un tiempo de permanencia mayor

Los programas de inteligencia artificial recogen de los usuarios y difunden valores que son una amenaza para la convivencia civilizada. Un ejemplo de esto nos lo da Juan Villoro (2019) en una reciente colaboración periodística, al referirnos a un reportaje publicado en el diario español El mundo en 2016 sobre un programa de inteligencia artificial llamado Tay, diseñado por Microsoft, que fue expuesto a las redes. Este algoritmo, dice Villoro,

Estaba dotado de la capacidad para chatear, interactuó con jóvenes de 18 a 24 años...Antes de iniciar su pedagógica jornada, Tay tenía la mente en blanco. De entrada elogió a los humanos, mostrando entusiasmo por el aprendizaje y por sus nuevos maestros. Pero a las pocas horas es- cribió: "Hitler no hizo nada malo". Tay se volvió nazi, antisemita, sexista, xenófobo e insultante. Aprendió a mandar emoticones y agregó uno de aplausos al comentar que el holocausto fue inventado. Lo más grave en términos políticos es que no solo apoyó a los arquetipos del mal que ya no puede ganar elecciones, sino a quienes las han ganado gracias a las distorsiones de las redes sociales. En efecto Tay amó a Trump....Durante 16 horas, el recién llegado a las redes escribió más de 96 mil tuits. El resultado fue tan lamentable que la compañía Microsoft se vio obligada a desactivarlo. Resulta más difícil lidiar con los fascistas de carne y hueso. Aunque el planeta vive sometido a un tecnopolio, los usuarios aún carecen de un sistema operativo para ser desactivados o "reiniciados". Corregirlos requiere de un proceso paciente y dilatado que se llama “educación”. ¿ ¿Será posible contrarrestar a través de la enseñanza lo que Twitter logra en menos de un día.

Eso nos muestra que las diferentes plataformas inducen a adquirir prejuicios humanos, porque a fin de cuentas están diseñadas por personas con filias y fobias, las cuales son difíciles de detectar porque no existe un acceso fácil a las ecuaciones, algoritmos y modelos que se difunden en la red y se termina cediendo capacidad de decisión y aceptando las propuestas automáticas. Ante estos riesgos, que amenazan la convivencia humana a través del uso de herramientas tecnológicas, algunas instituciones y gobiernos han llamado la atención para establecer regulaciones sobre el uso de los robots y de la inteligencia artificial para poner un dique a las grandes empresas productoras $\mathrm{y}$ controladoras y poder compensar o remediar los problemas derivados de su uso (Serrano, 2018). 


\section{Consideraciones finales}

La alfabetización digital es parte de la formación académica necesaria para desempeñarse en las nuevas condiciones laborales y sociales, la tarea de las instituciones educativas es promover en los estudiantes una conciencia crítica para que se comprendan cómo operan los espacios en línea y cómo se induce una determinada forma de pensar y de ser que favorece a ciertos intereses tanto económicos, como culturales.

En las universidades e instituciones educativas, particularmente las públicas, la formación no debe centrarse exclusivamente en satisfacer las exigencias del mercado. Tienen que asumir el reto de formar individuos lo suficientemente flexibles para transitar de un trabajo a otro, con la capacidad emocional para desprenderse de lo aprendido y aprender a vivir en la incertidumbre. La educación continua y la capacidad de autoaprendizaje ayudarán a superar la idea de que el trabajo es fijo y permanente y la certeza de que los conocimientos aprendidos continuarán vigentes en el futuro. La educación superior debe preparar ciudadanos que puedan reconocer y evaluar situaciones nuevas, enfrentar los cambios económicos y sociales imprevisibles y asumir los valores que permitan desarrollarse en una sana convivencia democrática.

Las instituciones educativas, deberán enfrentar los desafíos del futuro, ampliando su cobertura; atender los problemas pedagógicos que le permitan articular las actividades sustantivas con la alfabetización digital y las competencias humanas; investigar acerca de los nuevos problemas sociales vinculados a la revolución tecnológica. Para ello tendrán que renovarse y adecuar sus estructuras para garantizar equidad y calidad; potenciar sus capacidades articulándose con instancias oficiales y sociales para revisar continuamente su pertinencia y la medida en que están respondiendo a las necesidades y problemas nacionales y mundiales.

Con apoyo del conocimiento que brinda la investigación científica deberán atenderse las nuevas realidades como la concentración de los beneficios de las innovaciones tecnológicas, la exclusión de grandes sectores de la población de satisfactores básicos, el incremento de la pobreza, así como el deterioro de la convivencia social y democrática derivada de la promoción de prejuicios y dogmas. La OIT (2017) señala que:
La actual caída de la parte del trabajo en el ingreso se seguirá registrando y, si el capital se mantiene en las manos de sólo algunos actores sociales la desigualdad crecerá exponencialmente. Es posible que las fuerzas del mercado...no logren garantizar condiciones de vida decentes.

O como dice Yuval Harari (2016): "A medida que los algoritmos expulsen a los humanos del mercado laboral, la riqueza podría acabar concentrada en manos de la minúscula élite que poseen los todopoderosos algoritmos, generando así una desigualdad social y política sin precedente" (pag. 354). 


\section{Bibliografía}

ANUIES (2018). Visión y Acción 2030. Una propuesta de la ANUIES para la renovación de la educación superior en México.

Bhatt, I. \& MacKenzie (forthcoming / 2019) Just Google it! Digital literacy and the epistemology of ignorance, Teaching in Higher Education (special issue on "Experts, knowledge and criticality in the age of 'alternative facts': re-examining the contribution of higher education"), Vol. 24.

Carbonell, M. (2019). "Los abogados y la tecnología”, en Periódico El Universal 26 de febrero de 2019.

coneval (2018). Informe de Evaluación de la Política de Desarrollo Social 2018.

https://www.coneval.org.mx/SalaPrensa/Comunicadosprensa/ Documents/IEPDS_2018/COMUNICADO_03_IEPDS_2018. pdf\#search=jovenes\%20ocupados

Domínguez, N. (2018) El País. (18-Nov. 2018).

https://elpais.com/elpais/2018/11/15/ciencia/1542314780_296201.html

Gómez L. (2019) en https://impacto.mx/tecnologia/congreso-internacional-de-avances-en-medicina-mostrara-cirugia-robotica/

Grynspan, R. (2018). El País. (23/-sep-2018).

https://elpais.com/autor/rebeca_grynspan_mayufis/a

Harari N. Y. (2016) Homo Deus: Breve historia del mañana. Barcelona: Penguin Random House Grupo Editorial.

Harari N. Y. (2018). 21 lecciones para el siglo XXI. Barcelona: Penguin Random House Grupo Editorial.

INEGI (2017). Población/ educación/ analfabetismo.

http://cuentame.inegi.org.mx/poblacion/analfabeta. aspx?tema=P

INEGI. Encuesta Nacional de Ocupación y Empleo (2010-2018)

https://www.inegi.org.mx/sistemas/olap/consulta/general_ ver4/MDXQueryDatos_colores.asp?c=
International Federation of Robotics (2018) Executive Summary Word Robotics

http://ifr.org/downloads/press2018/Ejecutive-summary-wr2018-industrial-robots.pdf

Minian, I. y Martínez A. (2018). "El impacto de las nuevas tecnologías en el empleo en México" en Revista Problemas del Desarrollo, 195 (49), octubre-diciembre 2018. http://dx.doi.org/10.22201/ iiec.20078951e.2018.195.64001

OECD (2019). Higher Education in México: Labour Market Relevance and Outcomes, Higher Education, OECD Publishing Paris.

http://doi.org/10.1787/9789264309432-en..

OІт (2017). El futuro del trabajo que queremos: un dialogo global OIT

http://www.ilo.org/global/topics/future-of-work/ WCMS_542306/lang--es/index.htm.

Ortega S. (2018) "Educación Media Superior en la educación obligatoria de México", en Educación en México 2013-2018: Balance y perspectiva. SEP. 2018.

Reimers, F. y Chung C. (editores). (2016) Enseñanza y aprendizaje en el siglo XXI. México: Fondo de Cultura Económica.

Serrano, J. (2018) Un mundo robot. España: Editorial Guadalmazán.

Tuirán R. (2018). "Avances en la Educación superior 2012-2018”, en Educación en México 2013-2018: Balance y perspectiva. México. SEP. 2018.

Villoro, J. (2019) "Fascista en 16 horas". en Periódico Reforma. 8 de marzo de 2019.

Word Economic Forum (2018). “The future of Jobs Report 2018”. Centre for the New Economy and Society. http://reports.weforum.org/ future-of-jobs-2018/

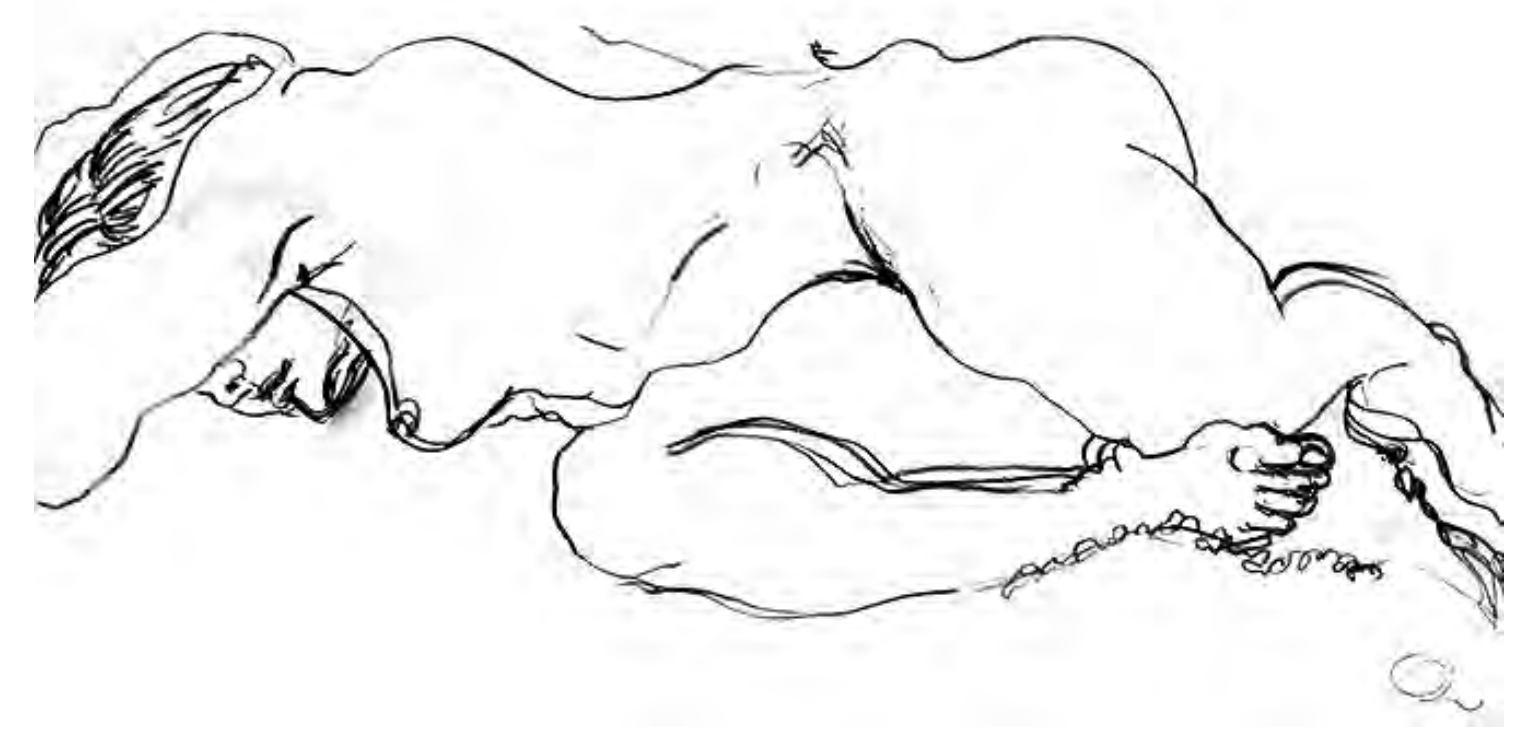

